\title{
The Traditional Japanese Formula Keishibukuryogan Inhibits the Production of Inflammatory Cytokines by Dermal Endothelial Cells
}

\author{
Yoko Yoshihisa, Megumi Furuichi, Mati Ur Rehman, Chieko Ueda, \\ Teruhiko Makino, and Tadamichi Shimizu \\ Department of Dermatology, Graduate School of Medicine and Pharmaceutical Sciences, University of Toyama, Sugitani, \\ Toyama 930-0194, Japan \\ Correspondence should be addressed to Tadamichi Shimizu, shimizut@med.u-toyama.ac.jp
}

Received 29 September 2010; Revised 11 November 2010; Accepted 12 November 2010

Academic Editor: Fulvio D’Acquisto

Copyright () 2010 Yoko Yoshihisa et al. This is an open access article distributed under the Creative Commons Attribution License, which permits unrestricted use, distribution, and reproduction in any medium, provided the original work is properly cited.

\begin{abstract}
Keishibukuryogan (KBG) is one of the traditional herbal formulations widely administered to patients with blood stagnation for improving blood circulation; currently, it is the most frequently prescribed medicine in Japan. KBG has been reported to improve conjunctional microcirculation. The aim of this study was to evaluate the role of KBG and paeoniflorin, a bioactive compound of KBG, in inhibiting the production of inflammatory cytokines using human dermal microvessel endothelial cells (HDMECs). The authors observed that lipopolysaccharide (LPS; $1 \mu \mathrm{g} / \mathrm{mL}$ ) stimulated the secretion of proinflammatory cytokines in HDMECs. KBG treatment $(10 \mathrm{mg} / \mathrm{mL})$ significantly suppressed the mRNA levels of migration inhibitory factor (MIF), interleukin (IL)-6, IL8 , and tumor necrosis factor (TNF)- $\alpha$ in LPS-stimulated cultured HDMECs. Similarly, paeoniflorin significantly suppressed the mRNA levels of these cytokines in LPS-stimulated cultured HDMECs. ELISA showed that KBG and paeoniflorin suppressed the production of MIF, IL-6, IL-8, and TNF- $\alpha$ in LPS-stimulated HDMECs. Moreover, KBG and paeoniflorin decreased the expression of cyclooxygenase-2 and inducible nitric oxide synthase (iNOS) in these cells. These results suggest that KBG may be useful for improving microvascular inflammation in patients with skin diseases.
\end{abstract}

\section{Introduction}

Keishibukuryogan (KBG, Gui-zhi-fu-ling-wan in Chinese) is one of the Kampo medicines, and has been widely administered to patients with blood stagnation for improving blood circulation. KBG is now one of the most frequently used medicines in Japan. KBG has been used clinically to treat various diseases, including skin diseases. It was reported that KBG improves conjunctional microcirculation in patients with cerebrospinal vascular diseases [1], thus suggesting that it may have beneficial effects on hematological parameters such as blood viscosity and red blood cell deformability [2-4]. Moreover, Matsumoto et al. have explored a proteomic approach for the diagnosis of blood stasis in rheumatoid arthritis patients treated with KBG [5]. In addition, KBG is used to treat symptoms of peripheral ischemia such as cold extremities [6]. Furthermore, we recently reported that KBG is effective in patients with chronic pigmented purpura, a group of skin vascular disorders of unknown etiology [7].

KBG is composed of five medicinal plants, Cinnamomum cassia Blume (Cinnamomi cortex), Paeonia lactiflora Pallas (Paeoniae Radix), Paeonia suffruticosa Andrews (Moutan cortex), Prunus persica Batsch (Persicae semen) and Poria cocos Wolf (Hoelen) (Table 1) [8]. Paeoniae Radix and Moutan Cortex have many known active components in common, including paeoniflorin, paeonol, oxypaeoniflorin, benzoylpaeoniflorin, and palbinone [9]. Paeoniflorin is a characteristic main principal bioactive component of Paeoniae Radix, which included approximately $5.57 \%(\mathrm{w} / \mathrm{w})$ paeoniflorin, and Moutan Cortex, which included approximately 3.96\% (w/w) paeoniflorin [10]. Paeoniflorin has been reported to have many pharmacological effects, such as anti-inflammatory and antiallergic effects [11]. Recently, Zheng and Wei reported that the total glucosides present 
TABLE 1: Components of keishibukuryogan (KBG).

\begin{tabular}{lccc}
\hline Japanese name & Scientific name & Botanical name & Ratio $(\mathrm{g})$ \\
\hline Keihi & Cinnamomi cortex & $\begin{array}{c}\text { Cinnamomum } \\
\text { cassia Blume } \\
\text { Paeonia }\end{array}$ & 1 \\
Tounin & Paeoniae radix & $\begin{array}{c}\text { Pactiflora Pallas } \\
\text { Persicae semen }\end{array}$ & $\begin{array}{c}\text { Prunus persica } \\
\text { Batsch }\end{array}$ \\
Bukuryou & Hoelen & $\begin{array}{c}\text { Poria cocos Wolf } \\
\text { Paeonia }\end{array}$ & 1 \\
Botanpi & Moutan cortex & $\begin{array}{c}\text { suffruticosa } \\
\text { Andrews }\end{array}$ & 1 \\
\hline
\end{tabular}

in the Moutan Cortex, which contain paeoniflorin as the principle bioactive component, inhibited primary and secondary inflammation in both collagen-induced arthritis and adjuvant-induced arthritis [12].

Up until now, topical and oral corticosteroids, oral bioflavonoids, ascorbic acid, griseofulvin, and cyclosporine have been suggested as treatments for chronic pigmented purpura $[13,14]$. However, none of these treatments have proven to be satisfactory. Human dermal microvessel endothelial cells (HDMECs) are the prominent cells in dermal skin. HDMECs produce inflammatory cytokines, such as interleukin (IL)-6 and IL-8 when they are exposed to lipopolysaccharide (LPS). We therefore consider that examinations of the effects of KBG and paeoniflorin on HDMECs are important for the therapeutic studies of chronic pigmented purpura in vitro.

In this study, we aimed to evaluate the role of KBG and paeoniflorin, ininhibiting the production of inflammatory cytokines using HDMECs.

\section{Materials and Methods}

2.1. Materials. KBG (TJ-25) was obtained from Tsumura \& Co. (Tokyo, Japan). KBG was suspended in CS-C complete medium containing $10 \%$ fetal calf serum and $1 \%$ penicillin and CSC growth factor (Cell Systems Inc, WA) and was rotated at $4^{\circ} \mathrm{C}$ overnight [11]. Then, the suspension was centrifuged, and the supernatant was filtered through a $0.45 \mu \mathrm{m}$-pore membrane. The following materials were obtained from commercial sources: the Isogen RNA extraction kit was obtained from Nippon Gene (Tokyo, Japan); M-MLV reverse transcriptase was from GIBCO (Grand Island, NY); Taq DNA polymerase was from Perkin-Elmer (Norwalk, CO); LPS was purchased from Sigma (St. Louis); nylon membranes were from Schleicher \& Schuell (Keene, $\mathrm{NH}$ ); the anticyclooxygenase-2 (COX-2) polyclonal antibody $(\mathrm{pAb})$ was purchased from Cell Signaling Technology, Inc. (Boston); antiinducible NOS (iNOS) pAb was purchased from Enzo Life Sciences International Inc. (NY); the anti- $\beta$ actin $\mathrm{Ab}$ was purchased from Santa Cruz Biotechnology Inc. (CA); Paeoniflorin was from LKT Laboratories, Inc.; MIF, IL-6, and IL-8 ELISA kits were obtained from R\&D Systems
(Minneapolis); and the Western blot detection system was obtained from Cell Signaling Technology (Beverly, MA). All other reagents were of analytical grade.

2.2. Cell Stimulation. Primary human dermal endothelial cells (HDMECs) were obtained from Cell Systems Inc (WA). HDMECs were cultivated in conditioned endothelial culture medium (CS-C complete medium) containing $10 \%$ fetal calf serum and $1 \%$ penicillin and CSC growth factor at $37^{\circ} \mathrm{C}$ in a $5 \% \mathrm{CO}_{2}$ atmosphere. Cell viability before treatment was always over $95 \%$ as evaluated by Trypan blue dye exclusion test. On the day of the experiment, cells were collected and suspended in fresh culture medium at a concentration of $1 \times 10^{6} \mathrm{cells} / \mathrm{mL}$. The cells were divided into 4 groups: a control group, a group receiving $10 \mathrm{mg} / \mathrm{mL}$ of $\mathrm{KBG}$ or $100 \mu \mathrm{g} / \mathrm{mL}$ of paeoniflorin as previously described $[9,15]$, a group treated with $1 \mu \mathrm{g} / \mathrm{mL}$ of LPS, and another group treated with the combination. The cells were treated with the various agents, and analyses were performed as described for each procedure.

2.3. MTT Assay. The number of cells was measured by an MTT assay. In brief, the media were removed by aspiration, and then the cells were treated with $0.5 \mathrm{mg} / \mathrm{mL}$ dimethylthiazol-2-yl-2,5-diphenyltetrazolium bromide (MTT, Sigma) in culture medium for 4 hours at $37^{\circ} \mathrm{C}$. After being washed with PBS once, isopropanol/ $/ 0.04 \mathrm{M} \mathrm{HCl}$ was added, and OD570 was measured, and the value of blank was subtracted.

2.4. Reverse Transcription-PCR Analysis. Total RNA was extracted from each mouse skin specimen. RNA reverse transcription was performed with M-MLV reverse transcriptase using random hexamer primers, and subsequent amplification was done using Taq DNA polymerase. PCR was carried out for 30 cycles with denaturation at $94^{\circ} \mathrm{C}$ for 30 seconds, annealing from 52 to $64^{\circ} \mathrm{C}$ for 1 minute and extension at $72^{\circ} \mathrm{C}$ for 30 seconds using a thermal cycler (PE Applied Biosystems Gene Amp PCR system 9700). The human MIF primers used were $5^{\prime}$-ATGCCGATGTTCATCGTAAAC-3' (forward) and 5'-GGCGAAGGTGGAGTTGTTCCA-3' (reverse). The IL-6 primers used were $5^{\prime}$-GATGCAATAACCACCCCTGACCC-3' (forward) and 5' -CAATCTGAGGTGCCCATGCTAC-3' (reverse). The IL-8 primers used were $5^{\prime}$-CATGACTTCCAAGCTGGCCGTG-3' (forward), $5^{\prime}$-CCACTCTCAATCACTCTCAGTTC-3' (reverse) [16]. The TNF- $\alpha$ primers used were 5 -ACACCGTCAGCCGATTTGC-3' (forward) and 5'-CCCTGAGCCATAATCCCCTT- $3^{\prime}$ (reverse). GAPDH was used as a positive control. The primers used were $5^{\prime}$-ACCCAGAAGACTGTGGAT- $3^{\prime}$ (forward) and $5^{\prime}$-TCGTTGAGGGCAATGCCA-3' (reverse). After PCR, the amplified products were analyzed using 2\% agarose gel electrophoresis.

2.5. Cytokine Release Measurements. Supernatants were collected after $24 \mathrm{~h}$ of incubation with LPS $(1 \mu \mathrm{g} / \mathrm{mL})$ and/or $\mathrm{KBG}(10 \mathrm{mg} / \mathrm{mL})$ and paeoniflorin $(100 \mu \mathrm{g} / \mathrm{mL})$. MIF, IL-6, and IL- 8 were assayed by ELISA, according to the manufacturer's instructions. The absorbance was measured with a microplate reader (Labsystems Multiskan Bichromatic). 
2.6. Western Blot Analysis. Cells were collected and washed with cold PBS. The cells were lysed at a density of $1 \times 10^{6}$ cells $/ 50 \mu \mathrm{L}$ of RIPA buffer ( $1 \mathrm{M}$ Tris-HCA, $5 \mathrm{M} \mathrm{NaCl}$, $1 \%$ Nonidet P-40 (v/v), 1\% sodium deoxycholate, 0.05\% SDS, $1 \mathrm{mM}$ phenylmethyl sulfonyl fluoride) for 20 minutes. After brief sonication, the lysates were centrifuged at $12,000 \mathrm{rpm}$ for 10 minutes at $4^{\circ} \mathrm{C}$, and the protein content in the supernatants was measured using a bio-rad protein assay kit (Bio-Rad, Hercles, CA). The protein lysates were denatured at $96^{\circ} \mathrm{C}$ for $5 \mathrm{~min}$ after mixing with $5 \mu \mathrm{L}$ of sodium dodecylsulfate (SDS) loading buffer, applied on an SDS polyacrylamide gel for electrophoresis, and transferred to nitrocellulose membranes. Western blot analysis was carried out to detect the expression levels of COX-2 and iNOS using specific antibodies [17]. Band signals were visualized on X-ray film using chemiluminescence ECL detection reagents (Amersham Biosciences, Buckinghamshire, UK). The relative amounts of proteins associated with specific antibodies were normalized according to the intensities of $\beta$ actin.

2.7. Statistical Analysis. The values are expressed as the means \pm SD of the respective test or control group. Statistically significant differences in the stimulation with LPS in the groups treated with KBG or paeoniflorin were evaluated by the nonparametric Mann-Whitney $U$ test. $P$ values of $<.05$ were considered to be statistically significant.

\section{Results}

3.1. The Effects of KBG on LPS-Stimulated Induction of Cytokines in HDMECs. LPS plays a fundamental role in the pathogenesis of a number of inflammatory diseases by inducing a distinctive pattern of inflammatory cytokine release. We used LPS to produce inflammatory conditions in HDMECs. The cells were incubated with or without $1 \mu \mathrm{g} / \mathrm{mL}$ of LPS or $10 \mathrm{mg} / \mathrm{mL}$ of KBG for 6 or $24 \mathrm{~h}$, and the cell viability was assessed. None of the treatments elicited cytoxicity in the cells at the tested concentrations and incubation times (Figure 1(a)). We examined the anti-inflammatory effects of KBG on the induction of inflammatory cytokines by LPS stimulation. LPS-induced protein levels of MIF, IL6 , IL- 8 , and TNF- $\alpha$ were significantly inhibited by KBG (Figure 1(b)). Treatment of the cells with $1 \mu \mathrm{g} / \mathrm{mL}$ of LPS increased in mRNA levels of these cytokines from $6 \mathrm{~h}$ after stimulation, but these mRNA levels were decreased by KBG (Figure 1(c)).

3.2. Effect of Paeoniflorin on LPS-Stimulated Induction of Cytokines in HDMECs. The cells were incubated with or without $1 \mu \mathrm{g} / \mathrm{mL}$ of LPS or $100 \mu \mathrm{g} / \mathrm{mL}$ of paeoniflorin for 6 or $24 \mathrm{~h}$, and the cell viability was assessed. Figure 2(a) reveals that the incubation with paeoniflorin did not elicit toxicity in cells at the tested concentration at either incubation time. LPS-induced protein levels of MIF, IL-6, IL-8, and TNF- $\alpha$ were significantly inhibited by paeoniflorin, and the inhibitory effect was stronger than that of KBG (Figure 2(b)). Moreover, to examine the effects of paeoniflorin on the mRNA levels of inflammatory cytokines, cells were treated with LPS for $6 \mathrm{~h}$ in the presence of $100 \mu \mathrm{g} / \mathrm{mL}$ of paeoniflorin. The LPS-induced MIF, IL-6, IL-8, and TNF- $\alpha$ mRNA levels were decreased by paeoniflorin (Figure 2(c)).

3.3. Inhibition of COX-2 and iNOS Protein Expression by Paeoniflorin. Next, we examined whether paeoniflorin could inhibit the production of COX-2 and iNOS. Western blot analyses revealed that the expression of COX-2 and iNOS was increased by LPS stimulation in HDMECs whereas the treatment with paeoniflorin resulted in a decrease in COX-2 and iNOS expression (Figure 3).

\section{Discussion}

Among traditional Japanese medicinal compounds, Kampo formulas originated from ancient Chinese medicine, and are currently recognized and reimbursed by the Health Ministry in Japan for the treatment of a wide variety of conditions. The Kampo formula KBG is frequently used in traditional Japanese and Chinese medicine to treat several symptoms, including skin diseases. The preparation has demonstrated anti-inflammatory and free-radical scavenging effects.

In the present study, we have demonstrated that KBG treatment significantly suppressed the protein and mRNA levels of MIF, IL-6, IL-8, and TNF- $\alpha$ in LPS-stimulated cultured HDMECs. Chronic pigmented purpura is a disease complex characterized by capillaritis and lymphocytic capillary damage in the papillary dermis. Several lines of evidence suggest the involvement of humoral or cellular immunity, as well as hemostasis, in the pathogenesis of this disease [18]. These patients are sometimes resistant to standard treatments, such as topical corticosteroids. Indeed, our previous data support the effectiveness of KBG, which led to a significant improvement in patients with chronic pigmented purpura [7]. It is reported that KBG can prevent the progression of atherosclerosis [19], and preserve vascular endothelial function in cholesterol-fed rabbits [19] and in hypertensive rats [20]. Furthermore, in diabetic rats, it was demonstrated to have protective effects against vascular injury [21], and to delay the development of diabetic kidney disease [22, 23]. Moreover, KBG has a favorable effect on impaired glucose metabolism in type 2 diabetes by improving glucose intolerance, and it has been suggested that some of this effect is derived from the reduction of TNF- $\alpha$ content in skeletal muscle [24]. Accordingly, KBG may exert beneficial effects that result in the inhibition of inflammatory cytokines in HDMECs. It was recently reported that MIF induces the endothelial expression of IL-8, thus resulting in leukocyte recruitment [25]. Since MIF is an initiator of other proinflammatory cytokines such as IL- 6 , IL- 8 , and TNF- $\alpha$, and regulates the induction of these cytokines [26, 27], we hypothesized that KBG may inhibit MIF-regulated inflammatory mediators.

We also demonstrated that paeoniflorin, a bioactive compound contained in KBG, suppressed the mRNA levels of these inflammatory cytokines in LPS-stimulated cultured HDMECs, similar to the effects of KBG. It is known that paeoniflorin has anti-inflammatory and antiallergic activities 

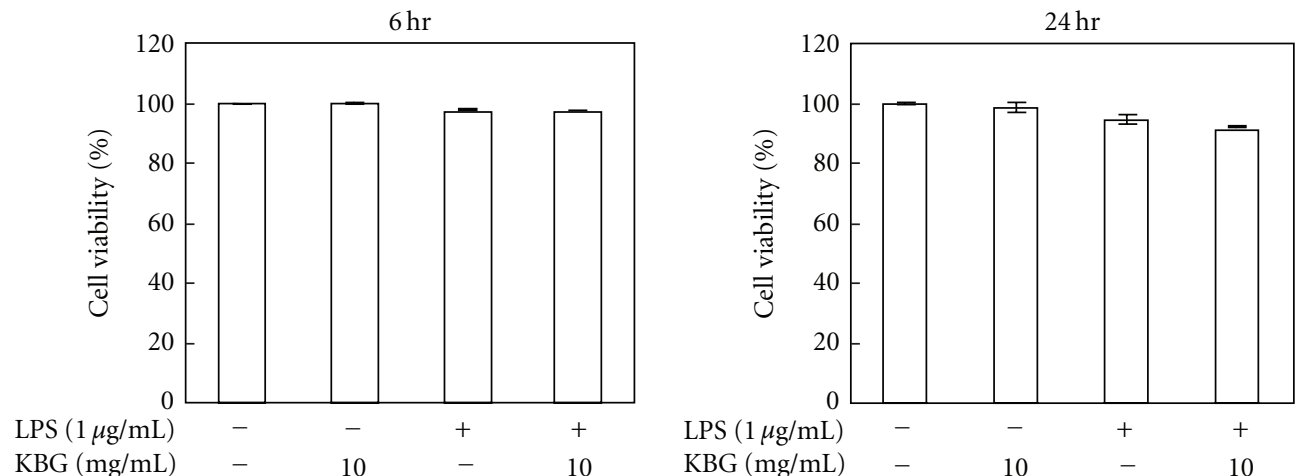

(a)
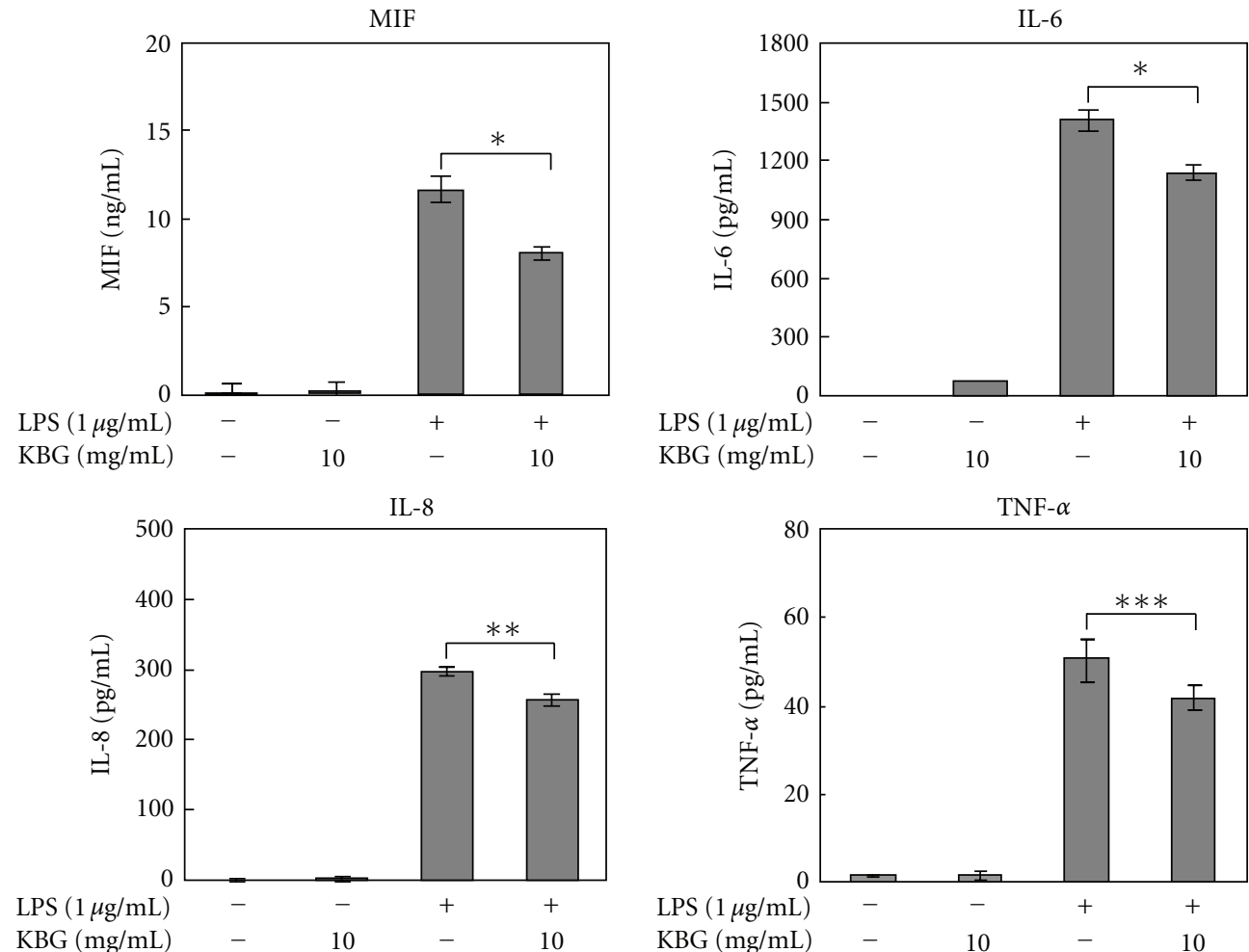

(b)

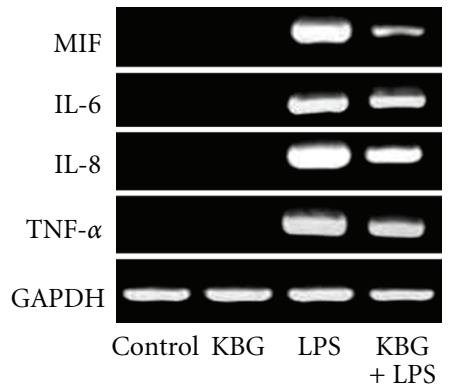

(c)

FIGURE 1: Effect of KBG on LPS-stimulated induction of cytokines in HDMECs. (a) HDMEC cells were treated with $1 \mu \mathrm{g} / \mathrm{mL}$ LPS and/or $\mathrm{KBG}(10 \mathrm{mg} / \mathrm{mL}$ ) for 6 or $24 \mathrm{~h}$ and were subjected to the MTT assay. (b) HDMEC cells were treated with $1 \mu \mathrm{g} / \mathrm{mL} \mathrm{LPS} \mathrm{and/or} 10 \mathrm{mg} / \mathrm{mL}$ of KBG for $24 \mathrm{~h}$. The MIF, IL-6, IL-8, and TNF- $\alpha$ content of cultured supernatants was analyzed by ELISA. Data are presented as the means \pm S.D $(n=5) .{ }^{*} P<.001,{ }^{* *} P<.005,{ }^{* *} P<.05$. (c) The cells were treated with $1 \mu \mathrm{g} / \mathrm{mL} \mathrm{LPS}$ and/or $10 \mathrm{mg} / \mathrm{mL}$ of $\mathrm{KBG}$ for $6 \mathrm{~h}$. Total RNA was isolated, and the mRNA expression levels of MIF, IL-6, IL-8, and TNF- $\alpha$ were detected by RT-PCR. (These experiments were repeated three times with similar results). 

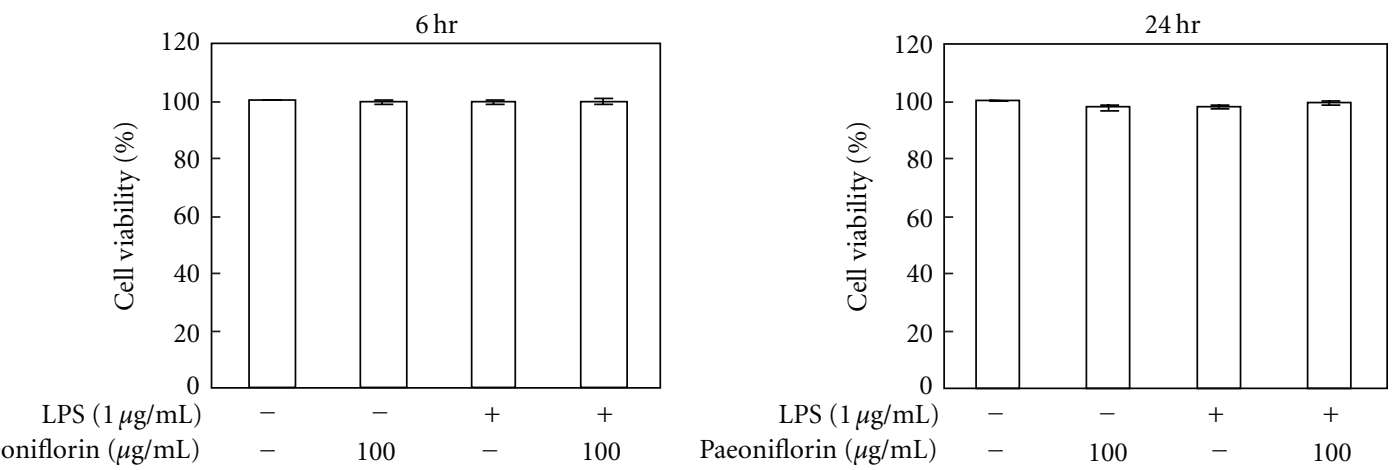

Paeoniflorin $(\mu \mathrm{g} / \mathrm{mL})$

100

Paeoniflorin $(\mu \mathrm{g} / \mathrm{mL})$

(a)
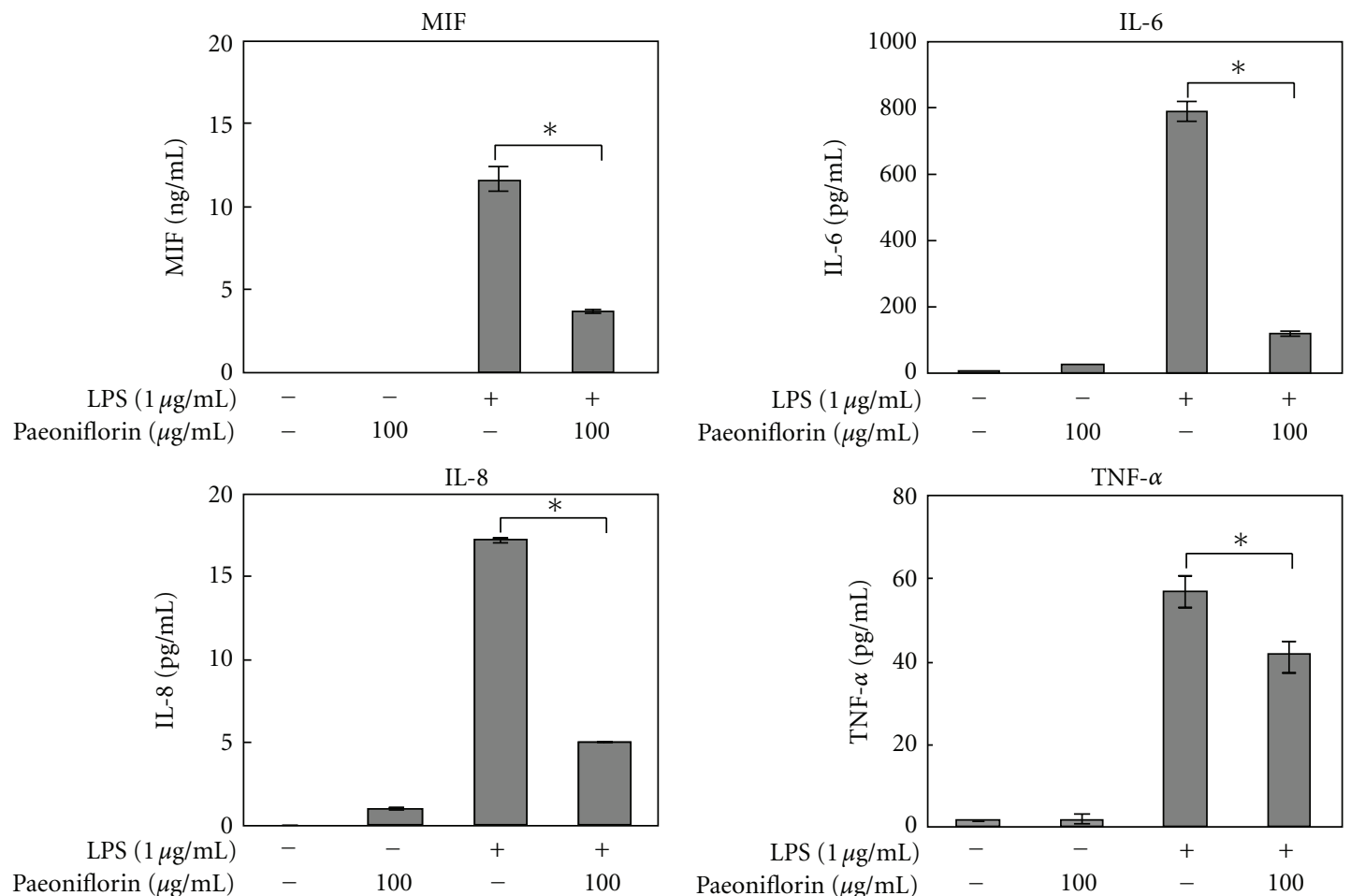

(b)

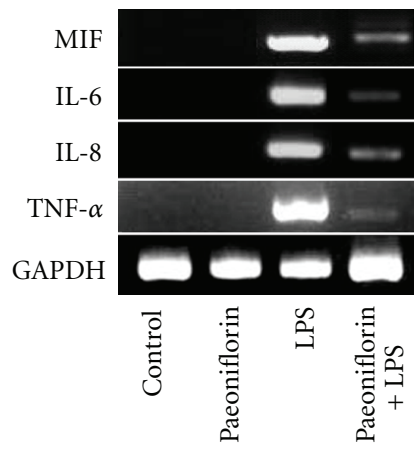

(c)

FIGURE 2: Effect of paeoniflorin on LPS-stimulated induction of cytokines in HDMECs. (a) HDMEC cells that were treated with LPS $(1 \mu \mathrm{g} / \mathrm{mL})$ and/or paeoniflorin $(100 \mu \mathrm{g} / \mathrm{mL})$ for 6 or $24 \mathrm{~h}$ and were subjected to the MTT assay. (b) HDMEC cells were treated with $1 \mu \mathrm{g} / \mathrm{mL}$ LPS and/or $100 \mu \mathrm{g} / \mathrm{mL}$ of paeoniflorin for $24 \mathrm{~h}$. The MIF, IL-6, IL-8, and TNF- $\alpha$ protein content of cultured supernatants was analyzed by ELISA. Data are presented as the means \pm S.D $(n=5) .{ }^{*} P<.0005$. (c) The cells were treated with $1 \mu \mathrm{g} / \mathrm{mL} \mathrm{LPS} \mathrm{and/or} 100 \mu \mathrm{g} / \mathrm{mL}$ of paeoniflorin for $6 \mathrm{~h}$. Total RNA was isolated, and the mRNA expression levels of MIF, IL-6, IL-8, and TNF- $\alpha$ were detected by RT-PCR. (These experiments were repeated three times with similar results). 


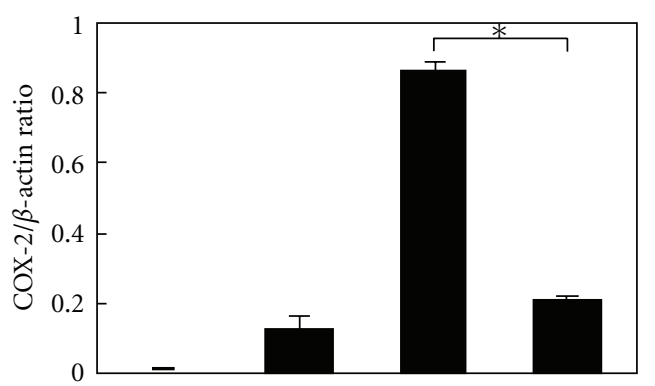

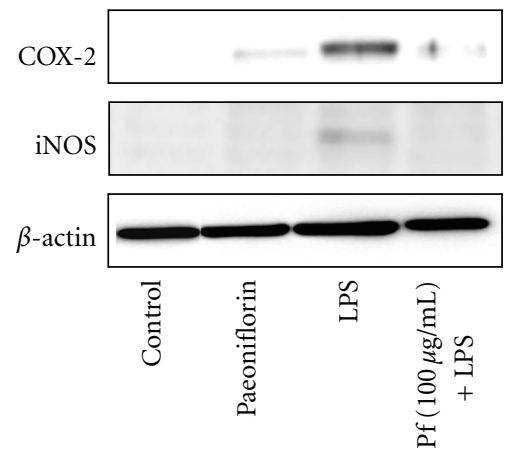

(a)

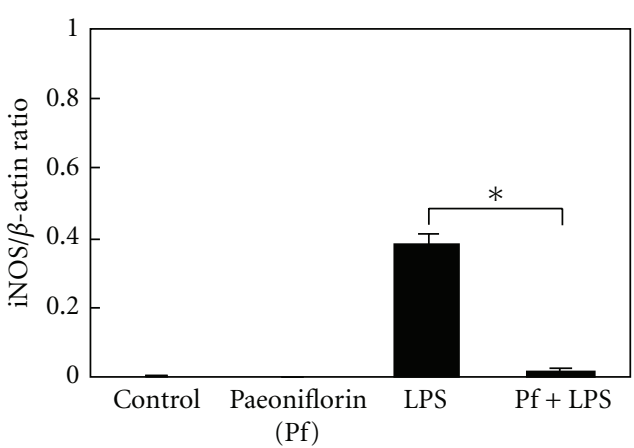

(b)

FIGURE 3: Effect of paeoniflorin COX-2 and iNOS protein expression. HDMEC cells were treated with $1 \mu \mathrm{g} / \mathrm{mL} \mathrm{LPS}$ and/or $100 \mu \mathrm{g} / \mathrm{mL}$ of paeoniflorin for $24 \mathrm{~h}$. COX-2 and iNOS protein expression in the cells was detected by a Western blot analysis, using $\beta$-actin as the internal control. The results of densitometric analysis, normalized with respect to $\beta$-actin using a bioimaging analyzer, are presented. Data are presented as the means \pm S.D $(n=5) .{ }^{*} P<.0001$. (This experiment was repeated three times with similar results).

$[28,29]$. In addition, paeoniflorin has reportedly exhibited immunoregulatory effects $[30,31]$, analgesic effects [32], neuromuscular blocking $[33,34]$, cognition enhancement [35], and steroid protein binding inhibition [36]. Our study revealed that paeoniflorin decreased the expression of COX2 and iNOS in HDMECs. Many studies have demonstrated that overproduction of nitric oxide and inflammatory prostaglandins such as PGE2, mainly via iNOS and COX2 , respectively, plays an important pathophysiological role in the development of inflammation [37]. Kim and Ha reported that paeoniflorin treatment significantly attenuated LPS-induced NO and PGE2 production [38]. Moreover, the extract of KBG has been shown to have COX-2 and inducible iNOS inhibitory activities [39]. In this context, we suggest that KBG and paeoniflorin induce their anti-inflammatory effects by inhibiting both proinflammatory cytokines and inhibiting the cascade of the overproduction of COX-2 and iNOS in HDMECs.

\section{Conclusion}

Taken altogether, these findings suggest that KBG may be useful to improve microvascular inflammation in patients with skin diseases such as chronic pigmented purpura.

\section{Nonstandard Abbreviations:}

ELISA: Enzyme-linked immunosorbent assay HDMECs: Human dermal microvessel endothelial cells KBG: $\quad$ Keishibukuryogan
IL: Interleukin

LPS: Lipopolysaccharide

MIF: Migration inhibitory factor

TNF: Tumor necrosis factor.

\section{Conflict of Interests}

The authors declare no conflicts of interest.

\section{Acknowledgment}

This research was supported by a Grant-in-Aid for Scientific Research (no. 20591337) from the Japan Society for the Promotion of Science.

\section{References}

[1] T. Itoh, K. Terasawa, K. Kohta, N. Shibahara, H. Tosa, and Y. Hiyama, "Effects of Keishi-bukuryo-gan and Trapidil on the microcirculation in patients with cerebro-spinal vascular disease," Journal of Medical and Pharmaceutical Society for WAKAN-YAKU, vol. 9, pp. 40-46, 1992.

[2] H. Hikiami, H. Goto, N. Sekiya et al., "Comparative efficacy of Keishi-bukuryo-gan and pentoxifylline on RBC deformability in patients with "oketsu" syndrome," Phytomedicine, vol. 10, no. 6-7, pp. 459-466, 2003.

[3] K. Kohta, H. Hikiami, Y. Shimada, H. Matsuda, T. Hamazaki, and K. Terasawa, "Effects of Keishi-bukuryo-gan on erythrocyte aggregability in patients with multiple old lacunar infarction," Journal of Medical and Pharmaceutical Society for WAKAN-YAKU, vol. 10, pp. 251-259, 1993. 
[4] H. Tosa, K. Toriizuka, and K. Terasawa, "The effect of Keishi-bukuryogan on blood viscosity and blood coagulation in normal subjects," Journal of Medical and Pharmaceutical Society for WAKAN-YAKU, vol. 4, pp. 172-179, 1987.

[5] C. Matsumoto, T. Kojima, K. Ogawa et al., "A proteomic approach for the diagnosis of 'Oketsu' (blood stasis), a pathophysiologic concept of Japanese traditional (Kampo) medicine," Evidence-Based Complementary and Alternative Medicine, vol. 5, no. 4, pp. 463-474, 2008.

[6] K. Fujita, T. Yamamoto, T. Kamezaki, and A. Matsumura, "Efficacy of keishibukuryogan, a traditional Japanese herbal medicine, in treating cold sensation and numbness after stroke: clinical improvement and skin temperature normalization in 22 stroke patients," Neurologia Medico-Chirurgica, vol. 50, no. 1, pp. 1-5, 2010.

[7] M. Furuichi, T. Makino, M. Yanagihara, and T. Shimizu, "Keishibukuryogan is effective in the treatment of chronic pigmented purpura," Science of Kampo Medicine, vol. 34, pp. 357-361, 2010 (Japanese).

[8] K. Nozaki, H. Hikiami, H. Goto, T. Nakagawa, N. Shibahara, and Y. Shimada, "Keishibukuryogan (Gui-Zhi-Fu-Ling-Wan), a Kampo formula, decreases disease activity and soluble vascular adhesion molecule-1 in patients with rheumatoid arthritis," Evidence-Based Complementary and Alternative Medicine, vol. 3, no. 3, pp. 359-364, 2006.

[9] H. Huang, E. J. Chang, Y. Lee, J. S. Kim, S. S. Kang, and H. H. Kim, "A genome-wide microarray analysis reveals anti-inflammatory target genes of paeonol in macrophages," Inflammation Research, vol. 57, no. 4, pp. 189-198, 2008.

[10] D. Z. Liu, K. Q. Xie, X. Q. Ji, Y. Ye, C. L. Jiang, and X. Z. Zhu, "Neuroprotective effect of paeoniflorin on cerebral ischemic rat by activating adenosine $A_{1}$ receptor in a manner different from its classical agonists," British Journal of Pharmacology, vol. 146, no. 4, pp. 604-611, 2005.

[11] J. Yamahara, T. Yamada, H. Kimura, T. Sawada, and H. Fujimura, "Biologically active principles of crude drugs. Antiallergic principles of "Shoseiryu-to". I. Effect on delayed-type allergy reaction," Yakugaku Zasshi, vol. 102, no. 9, pp. 881-886, 1982.

[12] Y. Q. Zheng and W. Wei, "Total glucosides of paeony suppresses adjuvant arthritis in rats and intervenes cytokinesignaling between different types of synoviocytes," International Immunopharmacology, vol. 5, no. 10, pp. 1560-1573, 2005.

[13] U. Reinhold, S. Seiter, S. Ugurel, and W. Tilgen, "Treatment of progressive pigmented purpura with oral bioflavonoids and ascorbic acid: an open pilot study in 3 patients," Journal of the American Academy of Dermatology, vol. 41, no. 2 I, pp. 207208, 1999.

[14] K. Okada, O. Ishikawa, and Y. Miyachi, "Purpura pigmentosa chronica successfully treated with oral cyclosporin A," British Journal of Dermatology, vol. 134, no. 1, pp. 180-181, 1996.

[15] T. Ara, Y. Maeda, Y. Fujinami, Y. Imamura, T. Hattori, and P. L. Wang, "Preventive effects of a Kampo medicine, Shosaikoto, on inflammatory responses in LPS-treated human gingival fibroblasts," Biological and Pharmaceutical Bulletin, vol. 31, no. 6, pp. 1141-1144, 2008.

[16] R. E. Unger, V. Krump-Konvalinkova, K. Peters, and C. J. Kirkpatrick, "In vitro expression of the endothelial phenotype: comparative study of primary isolated cells and cell lines, including the novel cell line HPMEC-ST1.6R," Microvascular Research, vol. 64, no. 3, pp. 384-397, 2002.

[17] J. J. An, S. Y. Kim, S. H. Lee et al., "Transduced PEP-1-Grb7 fusion protein suppressed LPS-induced COX-2 expression,"
Journal of Biochemistry and Molecular Biology, vol. 40, no. 2, pp. 189-195, 2007.

[18] I. Ghersetich, T. Lotti, S. Bacci, C. Comacchi, G. Campanile, and P. Romagnoli, "Cell infiltrate in progressive pigmented purpura (Schamberg's disease): immunophenotype, adhesion receptors, and intercellular relationships," International Journal of Dermatology, vol. 34, no. 12, pp. 846-850, 1995.

[19] N. Sekiya, H. Goto, K. Tazawa, S. Oida, Y. Shimada, and K. Terasawa, "Keishi-bukuryo-gan preserves the endothelium dependent relaxation of thoracic aorta in cholesterol-fed rabbit by limiting superoxide generation," Phytotherapy Research, vol. 16, no. 6, pp. 524-528, 2002.

[20] Y. Kasahara, H. Goto, Y. Shimada, N. Sekiya, Q. Yang, and K. Terasawa, "Effects of Keishi-bukuryo-gan (Gui-Zhi-Fu-LingWan) on endothelial function in spontaneously hypertensive rats," Journal of Traditional Medicines, vol. 18, pp. 113-118, 2001.

[21] H. Goto, Y. Shimada, N. Sekiya et al., "Effects of Keishibukuryo-gan on vascular function and hemorheological factors in spontaneously diabetic (WBN/kob) rats," Phytomedicine, vol. 11, no. 2-3, pp. 188-195, 2004.

[22] T. Nakagawa, T. Yokozawa, S. Oowada, H. Goto, N. Shibahara, and Y. Shimada, "Amelioration of kidney damage in spontaneously diabetic WBN/Kob rats after treatment with Keishibukuryo-gan," Journal of Traditional Medicines, vol. 20, pp. 156-164, 2003.

[23] T. Nakagawa, H. Goto, H. Hikiami, T. Yokozawa, N. Shibahara, and Y. Shimada, "Protective effects of keishibukuryogan on the kidney of spontaneously diabetic WBN/Kob rats," Journal of Ethnopharmacology, vol. 110, no. 2, pp. 311-317, 2007.

[24] T. Nakagawa, H. Goto, G. Hussein, H. Hikiami, N. Shibahara, and Y. Shimada, "Keishibukuryogan ameliorates glucose intolerance and hyperlipidemia in Otsuka Long-Evans Tokushima Fatty (OLETF) rats," Diabetes Research and Clinical Practice, vol. 80, no. 1, pp. 40-47, 2008.

[25] J. L. Gregory, M. T. Leech, J. R. David, Y. H. Yang, A. Dacumos, and M. J. Hickey, "Reduced leukocyte-endothelial cell interactions in the inflamed microcirculation of macrophage migration inhibitory factor-deficient mice," Arthritis and Rheumatism, vol. 50, no. 9, pp. 3023-3034, 2004.

[26] J. Nishihira, "Macrophage migration inhibitory factor (MIF): its essential role in the immune system and cell growth," Journal of Interferon and Cytokine Research, vol. 20, no. 9, pp. 751-762, 2000.

[27] T. Shimizu, H. Niizeki, O. Takeuchi et al., "Induction of macrophage migration inhibitory factor precedes the onset of acute tonsillitis," Mediators of Inflammation, vol. 13, no. 4, pp. 293-295, 2004.

[28] J. Yamahara, T. Yamada, H. Kimura, T. Sawada, and H. Fujimura, "Biologically active principles of crude drugs. II. Anti-allergic principles in "SHOSEIRYU-TO" antiinflammatory properties of paeoniflorin and its derivatives," Journal of Pharmacobio-Dynamics, vol. 5, no. 11, pp. 921-929, 1982.

[29] T. C. Chou, "Anti-inflammatory and analgesic effects of paeonol in carrageenan-evoked thermal hyperalgesia," British Journal of Pharmacology, vol. 139, no. 6, pp. 1146-1152, 2003.

[30] J. Liang, A. Zhou, M. Chen, and S. Xu, "Negatively regulatory effects of paeoniflorin on immune cells," European Journal of Pharmacology, vol. 183, no. 3, pp. 901-902, 1990.

[31] F. L. Hsu, C. W. Lai, and J. T. Cheng, "Antihyperglycemic effects of paeoniflorin and 8-debenzoylpaeoniflorin, glucosides from the root of Paeonia lactiflora," Planta Medica, vol. 63, no. 4, pp. 323-325, 1997. 
[32] E. Sugishita, S. Amagaya, and Y. Oghihara, "Studies on the combination of glycyrrhizae radix in shakuyakukanzo-to," Journal of Pharmacobio-Dynamics, vol. 7, no. 7, pp. 427-435, 1984.

[33] M. Kimura, I. Kimura, M. Muroi, T. Nakamura, and S. Shibata, "Depolarizing effects of glycyrrhizin-derivatives relating to the blend effects with paeoniflorin in mouse diaphragm muscle," Japanese Journal of Pharmacology, vol. 41, no. 2, pp. 263-265, 1986.

[34] K. Dezaki, I. Kimura, K. Miyahara, and M. Kimura, "Complementary effects of paeoniflorin and glycyrrhizin on intracellular $\mathrm{Ca}^{2+}$ mobilization in the nerve-stimulated skeletal muscle of mice," Japanese Journal of Pharmacology, vol. 69, no. 3, pp. 281-284, 1995.

[35] H. Ohta, J. W. Ni, K. Matsumoto, H. Watanabe, and M. Shimizu, "Peony and its major constituent paeoniflorin, improve radial maze performance impaired by scopolamine in rats," Pharmacology Biochemistry and Behavior, vol. 45, no. 3, pp. 719-723, 1993.

[36] T. Tamaya, S. Sato, and H. H. Okada, "Possible mechanism of steroid action of the plant herb extracts glycyrrhizin, glycyrrhetinic acid, and paeoniflorin: inhibition by plant herb extracts of steroid protein binding in the rabbit," American Journal of Obstetrics and Gynecology, vol. 155, no. 5, pp. 11341139, 1986.

[37] A. Ialenti, A. Ianaro, S. Moncada, and M. Di Rosa, "Modulation of acute inflammation by endogenous nitric oxide," European Journal of Pharmacology, vol. 211, no. 2, pp. 177$182,1992$.

[38] I. D. Kim and B. J. Ha, "Paeoniflorin protects RAW 264.7 macrophages from LPS-induced cytotoxicity and genotoxicity," Toxicology in Vitro, vol. 23, no. 6, pp. 1014-1019, 2009.

[39] C. H. Hong, S. K. Hur, O. J. Oh, S. S. Kim, K. A. Nam, and S. K. Lee, "Evaluation of natural products on inhibition of inducible cyclooxygenase (COX-2) and nitric oxide synthase (iNOS) in cultured mouse macrophage cells," Journal of Ethnopharmacology, vol. 83, no. 1-2, pp. 153-159, 2002. 


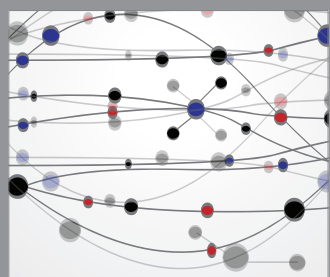

The Scientific World Journal
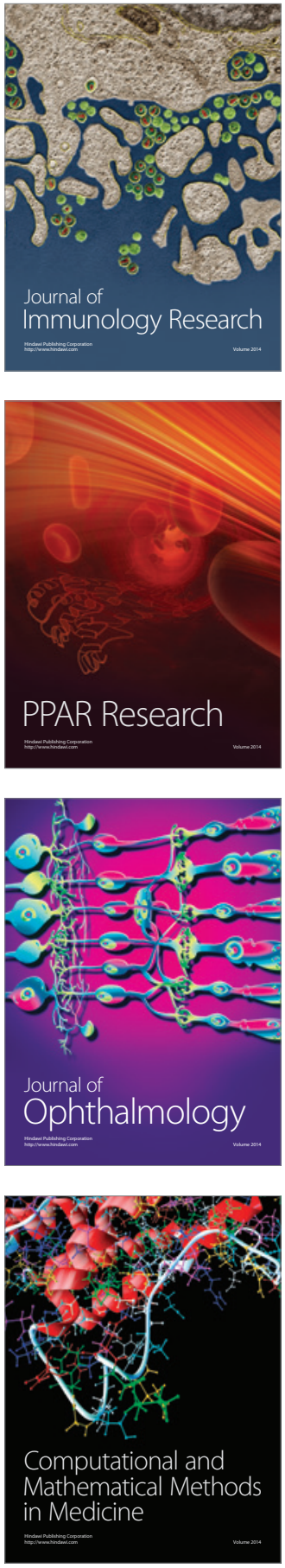

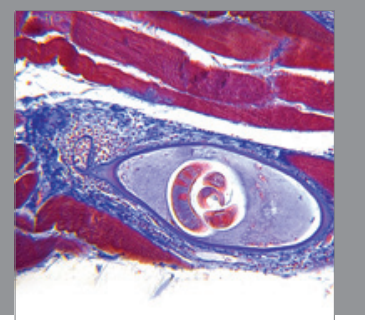

Gastroenterology

Research and Practice
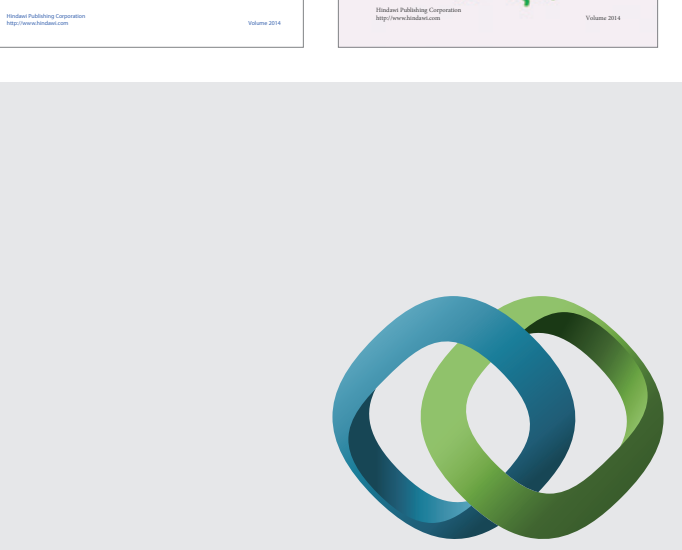

\section{Hindawi}

Submit your manuscripts at

http://www.hindawi.com
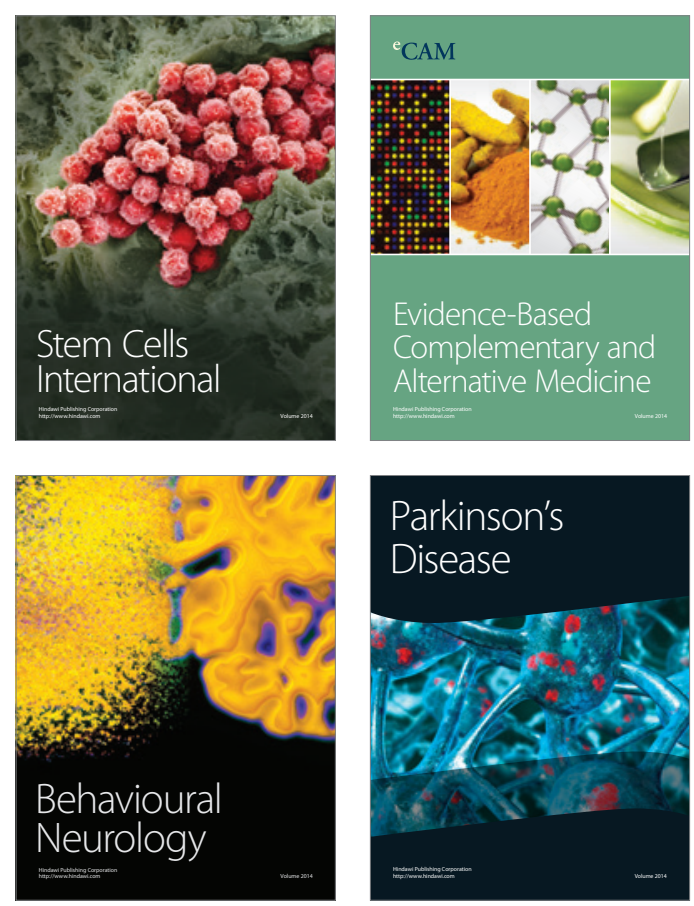

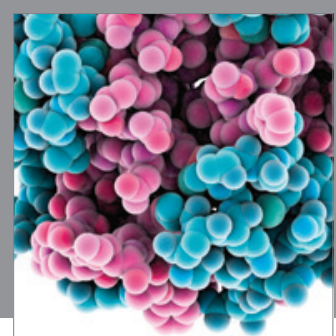

Journal of
Diabetes Research

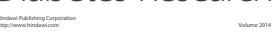

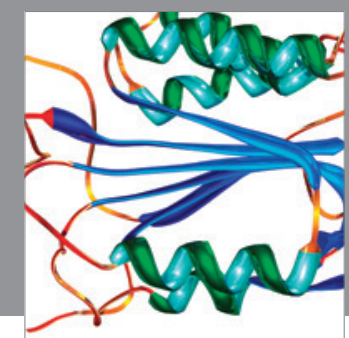

Disease Markers
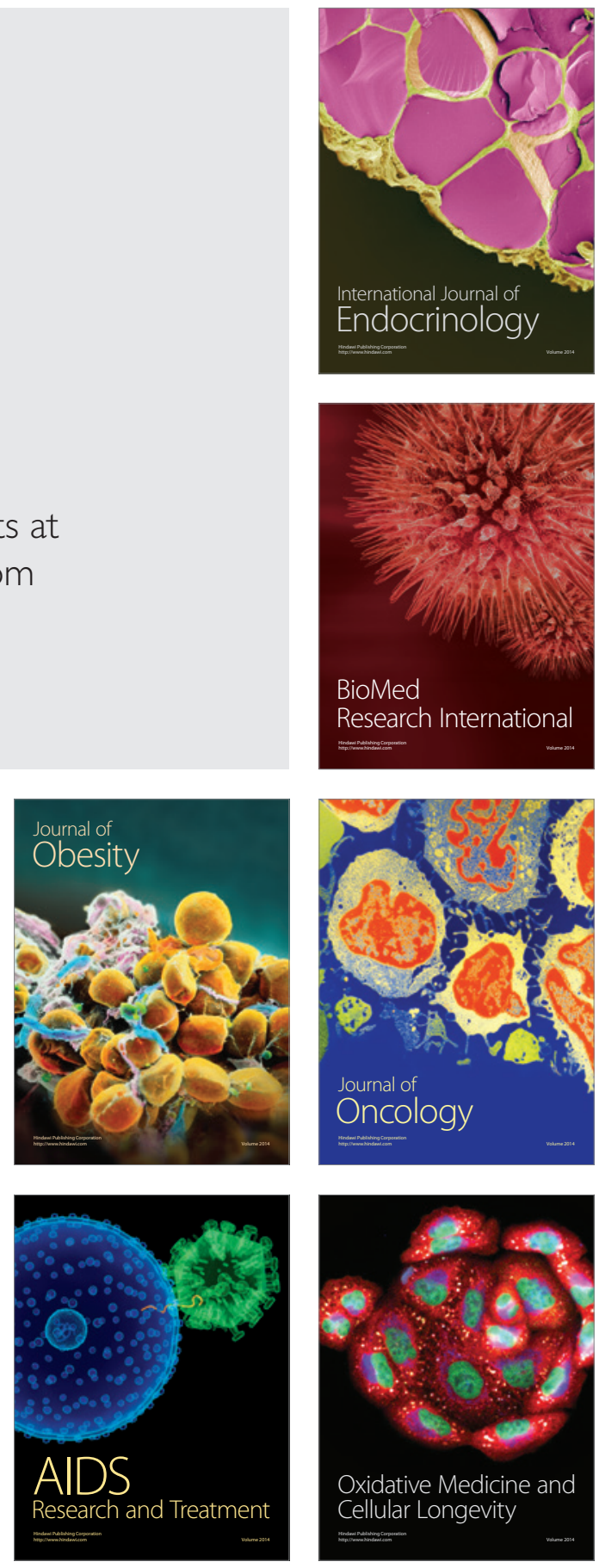